\title{
Smitizzazione e utopia in un romanzo di Giorgio Saviane
}

\section{Sandra Maria Boschetto}

Nell'ambito della narrativa italiana del dopoguerra Giorgio Saviane è uno scrittore atipico. Non già che manchino in lui elementi, stilistici e culturali, che possano essere stati mutuati da altri scrittorio dalle correnti letterarie piú affermate, ma il suo cammino si svolge solitario, ai margini della letteratura ufficiale, al di fuori delle gerarchie di valori stabilite. Del movimento detto neorealista, che si esaurí alla metà degli anni cinquanta (cioè prima che uscisse qualche romanzo di Saviane), rimangono nello scrittore solo alcune suggestioni ideologiche, di quell'ideologia antifascista che non costituiva la base. Del neorealismo gli mancano, invece, la volontà di scoprire l'Italia reale, nella sua arretratezza, nella sua miseria, nelle sue assurde contraddizioni, il gusto della rappresentazione fotografica, l'impasto linguistico di dialetto e lingua letteraria, l'amore per il folclore, la volontà di andare verso il popolo.

Saviane segue, invece, un'altra strada, con la quale, senza nascondersi o nascondere i mali della società contemporanea, cerca di elaborare, sul piano dell'arte, forme conoscitive che gli permettano di scavare in profondo, di valutare e "demistificare" la nuova realtà, ma nello stesso tempo di scoprire le correnti sotterranee che alimentano la lotta e la speranza degli uomini migliori. Come dice Carlo Salinari, "lo sperimentalismo di Saviane . . ha un fondamento diverso da quello neoavangardistico (e anche da quello precedente degli scrittori di Officina) perché legato al fascino dell'avventura, fondamento dell'umana vicenda: avventura che comporta necessariamente la proiezione verso l'ignoto e la sperimentazione di forme nuove ... ma che costituisce anche una proiezione al di sopra del dolore, della disperazione, della solitudine e della stessa gioia."1

L'ultima esperienza letteraria di maggiore svolgimento per l'autore è stata il romanzo, Eutanasia di un amore (1976). ${ }^{2}$ Come in precedenza con Il passo lungo (1965), sembra che Saviane abbia di nuovo cambiato direzione e, al posto di un romanzo d'idee, abbia voluto realizzare un romanzo d'amore, determinato dall'offesa profonda che la donna, Sena, ha subito quando Paolo l'ha costretta 
all'aborto, ferendola nelle viscere, nei suoi istinti vitali. Ancora una volta Saviane, senza alcun calcolo preconcetto, visto che la prima stesura del libro precede di molti mesi l'esplosione del problema sociale dell'aborto, sembra aver captato quasi nell'aria una questione importantissima per l'intera collettività. ${ }^{3}$ Tuttavia non è l'aborto l'argomento reale del libro, o, meglio, può anche essere l'aborto ma in una dimensione che non comprende le dispute di questi ultimi tempi.4

La prima chiave di lettura è quella del romanzo d'amore, un bel romanzo con molte pagine d'intensità di rappresentazione e di scrittura. Si conferma ancora una volta la caratteristica fondamentale dello stile e del linguaggio di Saviane, nel quale la comunicazione del messaggio ideologico avviene non attraverso un codice espressivo, logico o naturalistico, ma attraverso un linguaggio da sogno o da visione, con parole quasi pronunciate in trance, che della visione appunto hanno la lucidità e la chiaroveggenza, ma anche l'ambiguità, l'allusività, la mancanza di rispetto per le regole, il tono allucinato e fantastico, oratorio ed epico nello stesso tempo. 5 Il significante si collega, quindi, al significato.

$\mathrm{Vi}$ sono due personaggi che si alternano nella narrazione in forma diretta e indiretta, Paolo e Sena. L'inizio è ex abrupto e ci porta subito nel cuore della vicenda: una macchina si ferma al semaforo, una ragazza scende, una portiera sbatte. Paolo rimane solo. Fino a quel momento non aveva fatto che litigare. Ma ora il mondo sembra fermo, l'aria è spessa, "un meccanismo si è rotto con quella portiera sbattuta" (10). Raggiunge Sena, la prega di desistere dal suo atteggiamento almeno per non essere scortese con gli amici che li aspettano in campagna. Sena si piega, ma il suo profilo è durissimo: "Paolo sente di odiarla. Ma ha paura di quell'aria fuori, dei colori, del fosso di acqua sorgiva vicino alla loro casa, dei fiori: il verde soprattutto lo spaventa, la massa di verde dietro la fila dei cipressi: senza di lei il mondo si capovolgeva a ringhiare" (10-11).

La situazione è già delineata; qualcosa s'è introdotta nell'amore spensierato e stupefacente che legava i due amanti. Il disprezzo di Sena investe Paolo, che viene a trovarsi in una condizione oscillante fra momenti di insofferenza e la consapevolezza che non può vivere lontano dalla donna. Sena è la creatura femminile che Saviane ha sempre vagheggiato nella sua fantasia: bellissima ma anche spavalda, intraprendente, anticonformista, coraggiosa. L'unione fra lei e Paolo era stata libera, senza contratto matrimoniale, un amore senza impegno di perennità o di eterna fedeltà. Paolo, ora che Sena lo sfugge, ricorda ogni momento del loro amore, i bisticci, le sue tenere attenzioni; le preoccupazioni gelose (15). Non accetta la rottura, non ne comprende la ragione, si sente sconfitto e la cerca; vuole in tutti i modi incontrarsi con 
lei. Viene a sapere in quale istituto privato Sena insegna e va ad aspettarla all'uscita. È emozionato, è arriva to in anticipo, lo riempie la speranza di rivederla, finalmente. Ma un pensiero terribile gli attraversa la mente: se venisse un altro a prenderla? "Di fronte a una realtà probabile, l'unica che giustifichi il misterioso e improvviso fuggire di Sena, si sente scoperto, uno sciocco ometto che rincorre la ragazza che non lo vuole" (30). Diventa goffo. Quando Sena esce dalla scuola, imbacuccata per ripararsi dalla pioggia, non la riconosce. In Eutanasia di un amore, come in altri racconti di Saviane, c'è un'ispirazione umoristica, se si vuole dare all'umorismo il significato che gli attribuiva Pirandello di "sentimento del contrario," per cui l'umorista vanificava sempre ogni possibile illusione, mettendo in luce il suo contrario. La volontà beffarda di Saviane si diverte a farci vedere il rovescio della medaglia, deforma in smorfia non si sa se di riso o di pianto. ${ }^{6}$

Questo è Paolo che, dopo aver ferito profondamente Sena, dopo averla perduta, la rincorre disperatamente e sente che non puo vivere senza di lei. A lui si contrappone Sena. Le pagine in cui è lei ad agire e a parlare sono fra le più belle e intense del romanzo. Sena ha amato davvero Paolo; gli ha dato per dieci anni ogni minuto della sua vita. Ed è proprio per salvarli, per poterli avere sempre con sé che ella ha troncato i rapporti con lui. C'è stato un fatto che rende impossibile il loro amore. Un fatto che Paolo non potrebbe capire e magari ci riderebbe su o si difenderebbe con quella sua "vitalità teorica per cui può calpestare tutto avendo persino ragione" (70). Paolo è intelligente, sensibile, generoso, ma la sua "sete di nuovo, di riforma" (69) è più importante in lui di ogni altra cosa. Per questo preferisce lasciarlo, piuttosto che rivelargli il fatto che le impedisce di stargli vicino. Cosí accetta una borsa di studio a Parigi. ${ }^{7}$

Fin qui due storie parallele, anche se strettamente intrecciate. Paolo cerca Sena e questa lo sfugge. Entrambi, peró, si desiderano. Pagine ricche di un'analisi sottilissima degli stati d'animo contrastanti, delle incertezze, del volere e non volere. La costruzione narrativa è assai complessa, con l'alternanza del racconto in terza persona e quello più frequente della narrazione in prima persona dei due protagonisti. Ma soprattutto il lettore è tenuto sospeso, perché ormai sa che fra i due c'è un fatto che li divide e non è riuscito ancora a comprendere cosa sia. A Parigi avviene la prima svolta della vicenda. Paolo parte per raggiungere Sena, cerca di sapere, superando molti ostacoli, dove si tiene il corso di storia del cinema. Gli dicono che è a Versailles. Vi si trasferisce e intanto nella mente si affollano i ricordi di un precedente soggiorno in quella località e la fantasia anticipa con fervore il momento in cui rivedrà Sena e potrà abbracciarla. È felice. Si nasconde dietro una 
siepe per sorprenderla. Ma ecco che Sena sopraggiunge allacciata a un altro uomo, Domenico, un ingegnere edile che la bacia proprio a pochi passi da Paolo. È il crollo di tutto: quell'amore inimitabile sembra finito nel modo più banale e più ovvio. La donna ha un altro uomo. È l'antica legge del mondo, che l'amore sia legato al tradimento. Paolo perde il gusto di vivere: sembra che il suo corpo non si difenda piú contro il male. Una dopo l'altra l'affliggono varie malattie, che lo rendono quasi un rottame, quasi un vecchio. Alla fine s'incontra di nuovo con Sena, ritrovandola affettuosa come prima. Vanno insieme a Ischia, dove conoscono un personaggio singolare, il mago, vale a dire un dottore che cura i malati in modo diverso da tutti gli altri. Paolo ne ha un beneficio non solo fisico, ma anche spirituale. Le discussioni col mago aprono nuove prospettive alle sue idee. Finalmente, durante una gita in motoscafo, Sena rivolge a Paolo l'accusa che da mesi tiene dentro di sé, rivela la colpa che gli rimprovera. Paolo l'ha costretta ad abortire. Perché non vuole un figlio da lei? Si ricrea l'abisso fra di loro. Le ragioni di Sena sono quelle dell'istinto. La mancanza di un figlio per lei è una frustrazione. Le ragioni di Paolo sono quelle dei principi, dell'ideologia: "la famiglia è un istituto decrepito. Va cambiato" (143). Sena sarebbe stata un'ottima madre e avrebbe confermato il semplicismo familiare, imporre ai figli "il marchio del cognome, la parentela, i diritti e i doveri dell'amore" (144). Ma quella di Paolo non era un'ideologia di comodo: "Comodo sarebbe per me - egli dice - sposarti, unire i nostri stipendi, prendere una donna di servizio che ci educherebbe il pupo. Gli spaghetti li troverei sempre al dente. I figli mi darebbero qualche grana, si sa, ma molte soddisfazioni" (144). Ma tutto questo è possessivo ed egoistico, serve ai genitori non ai figli. All'amore animale o, naturale, Paolo vuol sostituire un amore culturale che capovolga il possessivismo del primo in altruismo e che intenda modificare il rapporto che è all'origine dell'amore, quello tra madre e figlio. Ecco la seconda chiave di lettura del libro, che rimane sempre un romanzo d'amore, $\mathrm{ma}$ in una dimensione tutta diversa. I due piani sono, naturalmente, intrecciati fra loro. Basta rilevare con quanta frequenza nella mente di Paolo, inconsapevolmente, Sena si confonda con l'immagine della madre: "Una sera, che lei era dovuta partire lasciandolo nella camera dell'albergo fitta della fragranza del suo corpo, aveva sospettato di un meccanismo riferibile all'infanzia, il corpo di sua madre onnipresente" (14). E ancora. Egli corre alla ricerca di Sena e le strade di Firenze sembrano confluire nella piazza del suo paese, quando lui, bambino, correva a cercare la madre che aveva perduto durante la musica in piazza. Sena è la grande madre di tutti, il mare: "Senza 
di lei ero come le tartarughe d'acqua che escono dall'uovo e vedono il mare e vorrebbero già esservi dentro, per sfuggire i rapaci. Ne arriverà una alle tenere acque, ma io non potevo essere quella, lei era il mare e la distanza dal mare e insieme la copertura di quella distanza insanabile" (52). Madre è Sena quando s'intenerisce per il cappotto troppo largo, che lo ingobbisce, per la sua distrazione, per la sua ingenuità. Ma soprattutto quando la vede abbracciata a Domenico esplode in lui l'immagine di sua madre durante il parto che lo mise alla luce: "neanche mia madre sapeva che io non volevo nascere, lasciare il sicuro angolo inerte per la vita . . mia madre urlava disperatamente dalle labbra di Sena che baciavano Domenico, urlava a me perché lasciassi il suo corpo. Io non volevo abbandonare quell'acqua tiepida, e mani invisibili mi premevano inesorabilmente verso la chiusa che si sarebbe spalancata nell'ignoto"' (99).

Infine questi lampi, intuizioni, folgorazioni, trovano la loro chiarezza nel colloquio col mago d'Ischia. L'infatuazione per Sena equivale all'infatuazione per la madre. Ma del complesso materno ci si puó liberare. In realtà non abbiamo una madre sola: "madre è chiunque ci ami nel momento dopo la nascita. Una madre professionista, e forse più madri intercambiabili, renderebbero meno severo il complesso edipico" (131). Smitizzazione, quindi, dell'amore naturistico, della madre che non può più essere la madonna, smitizzata appunto in Sena-Silva. Le parole del mago hanno confermato Paolo in alcune sue profonde persuasioni. Egli comprende che anche la gelosia appartiene all'amore naturale. "Sena tocca a Paolo esclusivamente, come ogni madre per il figlio: questo il complesso da estirpare. Difficile perché sacro, perché denso di lacrime, di tenerezza, di consenso, di ammirazione. Come dire a uno, tua madre è la tua ansia se per ciascuno madre è adorazione? Come dire a se stesso: Sena non è tua, e adesso rappresenta l'amore a quello che le ha infilato l'accappatoio sul corpo nudo ... la mia mamma, la mia famiglia, la mia Sena, capovolgono l'amore" (151).

Queste riflessioni di Paolo sono la chiave di volta del romanzo. La chiave di volta sul piano narrativo perché, se la prima parte è dominata dalla ricerca di Paolo e dal sottrarsi di Sena, la seconda, invece, è dominata dalla volontà di Paolo di sottrarsi al fascino esclusivo di lei. La chiave di volta ideologica perché quelle riflessioni sono l'approdo reale del romanzo e da essa scaturisce anche la soluzione narrativa: un naufragio del loro motoscafo nel quale sembra che Sena sia morta. In tutti i libri di Saviane un incidente (di solito automobilistico) interviene quasi sempre come elemento fondamentale della trama. Cosí è, di fatti, nell'ultimo romanzo, 
anche se non automobilistico e non fatale. ${ }^{8} \mathrm{Ed}$ è in questo momento che le persuasioni culturali coincidono con le ragioni esistenziali. Paolo riesce a salvare Sena: la salva dalla morte fisica. Naturalmente, poiché l'ama ancora, vuole che viva. Ma non puó dimenticare (anche se ne è profondamente turbato) la gioia selvaggia che aveva provato, insieme al terrore, nel crederla morta. "Adesso che sapevo che sarebbe vissuta avrei potuto piangere una tenerezza improvvisa come hanno le madri per il figlio che nasce o che lascia la casa. Mi pareva che la vita, la sua e tutte le vite, fossero una povera cosa di fronte al ricordo di quella galleria sotterranea di liquidi tiepidi dentro al mare infuriato. Era una minaccia vivere" (164). In realtà l'amore è morto e il resto del racconto è la storia della fine dell'amore. L'occasione è una nuova donna Silva, con la quale Paolo rimane bloccato due giorni e due notti, dalla tempesta, nell'isola delle Formiche. Silva l'attrae, può in qualche modo sottrarlo all'esclusivo possesso di Sena. Soprattutto, ed è una sorta di rivelazione, è sterile. La donna sterile fa parte della natura e deve avere una sua funzione. Silva senza volerlo lo illumina: la funzione della donna sterile è quella di essere madre dei figli altrui. L'utopia di Paolo trova una conferma nella realtà: Silva vive nell'utopia. Il libro puo finire con la solitudine di Paolo e col saldarsi della sua utopia con la grande madre universale, il mare, madre "dei pesci, degli animali e degli angioli. Anche gli angioli vengono dal mare. Anche i terribili retori che affermano di essere stati creati a immagine e somiglianza di Dio onnipotente. Onnipotente di che? Di uccidere un amore perché ne sorga un altro uguale? Onnipotente è il dolore: mio e della materia messasi testardamente sulla strada della conoscenza, con Sena e il mare, a riproporre la mia nascita, freddamente" (211).

Come si vede una storia d'amore e anche d'idee. La vicenda della fine di un grande amore fa da supporto a una generosa utopia, a una città del sole, nella quale domina l'altruismo, l'amore del rapporto di amore-odio fra madre e figlio. Come spiega lo stesso Saviane, se l'a more è "ripetizione dell'amore materno, si riproduce nel rapporto uomo a uomo il rapporto tra madre e figlio (o figlia) e se la madre non avrà, e quindi non trasmetterà al figlio, amore possessivo, che significa amore geloso, violento, si avranno nuovi rapporti tra gli uomini improntati a un amore non più esclusivo, istintuale, protettivo: ma amore, che ridotto alla sua espressione fisica è, forse, la forza di attrazione gravitazionale."9 Utopia impopolare, certamente, perché tocca sentimenti radicati nell'essere dalla stratificazione di milioni di generazioni: e tuttavia coraggiosa e reale, perché mette a nudo aspirazioni profonde della personalità 
che, appunto, la tradizione, il conformismo, l'opinione comune cercano di coprire e di nascondere.

I romanzi di Saviane, sono tutti strutturati su due piani, quello della realtà e quello della visione, dall'essere al dover essere. Il distacco dall'amore naturale, per tendere a una forma diversa d'amore che sia solo altruismo, accresce la solitudine e il dolore. Ma Paolo sa che solo il nuovo tipo di amore può vincere la solitudine, perché essa può essere conosciuta e superata solo nell'altruismo senza riserve e senza residui narcisistici. Sena, rimane sul piano "naturale" e rifiuta di seguire Paolo nel mondo dell'utopia. "Come quando era morta mia madre e come quando ero nato e la cercavo. Quando morí, io non volevo che morisse. Ma doveva. Sena correva via sullo yacht, con Domenico. Ero proprio solo, ad accreditarmi nei confronti di me stesso, senza più madre" (210). Silva, che compare solo nell'ultima parte del libro, al contrario di Sena, vive tutto nella dimensione utopica. Per questo è meno scoperto, quasi enigmatico e il suo disegno è volutamente privo di tratti troppo netti e marcati. La madre dei figli degli altri è troppo proiettata nel futuro per avere una caratterizazione psicologica delineata senza chiaroscuri. Infine, il mago di Ischia: anch'egli vive con purezza una sua utopia, folgorante di intuizioni sconcertanti, fiduciosa nelle risorse della natura, vigile sugli impulsi del subconscio con una distaccata comprensione delle umane passioni. Muore improvvisamente: "un infarto fulminante dovuto al troppo prodigarsi. Sussurrano che non dicesse mai di no: né ai malati, né alle donne e neanche agli uomini, una specie di dolce Rasputin sempre pronto a guarire, a amare" (137).

"Eutanasia" di un amore, insomma: la storia della fine di un amore. Ma il romanzo vuol essere anche, e soprattutto, la storia dell'amore, vale a dire di quel fenomeno che - come dice lo stesso Saviane - ha popolato il mondo e forse l'universo, ma che per diventare veramente umano "deve rompere le pastoie dell'esclusivismo possessivo, il complesso dell'amore materno, e proiettarsi versi l'altro, verso tutti gli uomini, verso la società." 10

Lewis and Clark College

\section{NOTE}

1 Carlo Salinari, Il Castoro, 120 (dicembre 1976), 68.

2 Opere di Giorgio Saviane: Le due folle (Parma, 1957), con il nome di Federico Saviane; L'inquisito (Milano, 1961); Il Papa (Milano, 1963); Il passo lungo (Milano, 1965); Le molte giustizie, a cura di Giorgio Saviane (Milano, 1967); Il mare verticale (Milano, 1973); Di profilo si nasce (Milano, 1974); Eutanasia di un amore (Milano, 1976). Le pagine citate nel testo fra parentesi vengono prese dalla seconda edizione BUR: agosto 1979. Eutanasia ha vinto nel 1977 il XXV Premio Bancarella. Nel $1977 \mathrm{fu}$ anche portato allo schermo cinematografico. 
3 Cosí, di fatti, era avvenuto nel primo romanzo con la denuncia della violenza negli stadi, nel secondo a proposito della modifica del codice di Procedura penale, ne Il Papa (1963) con i fermenti del mondo cattolico. Ė necessario tener sempre presente che l'anticonformismo è un caposaldo della concezione del mondo di Saviane, nella quale ogni progresso è affidato al gesto ribelle, alla rottura della norma. Il Professore Gabriele-Aldo Bertozzi, docente della Università di Roma, in un incontro svoltosi nel giugno del 1980 a Roma riconferma la spinta filosofica e il fascino ideologico che continua a esercitare Saviane fra i giovani d'oggi, suoi più entusiasti lettori. Attualmente, Saviane sta scrivendo un nuovo romanzo, Getsèmani, basato sulla storia di Cristo.

4 Giuliano Manacorda, i Contemporanei (Marzorati, 1974), pp. 285-86, ha cercato di individuare le caratterristiche di questo stile: "Dal punto di vista morfologico e sintatico: 1) l'uso frequente di un periodare brevissimo, spesso ellitico del predicato, talora monoverbico; 2) l'inversione di soggetto e predicato; 3) lo scambio di verbi transitivi e intransitivi e l'uso dei verbi con la costruzione inusitata; 4) la soppressione dell'articolo; 5) alcune forzature ortografiche o invenzioni lessicali. Dal punto di vista stilistico; 6) l'uso frequentissimo della personificazione e quello un po' meno frequente dell'iperbole; 7) la elencazione generalmente in forma di climax; 8) l'anteposizione dell'aggettivo al sostantivo e le sinestesi; 9) la scelta di parole impreviste o impreviste in quella sede; 10) ma soprattutto un legame fra le immagini affidato più alla fantasia che alla logica, più all'invenzione che alla necessità.'

6 Vedasi L. Simonelli, "Lo scrittore che vive in mezzo a un harem," La Domenica del Corriere, 26 ottobre 1976. Anche le pagine dedicate a Saviane di Riccardo Tanturri, La Linea del Conformismo (Padova, 1973), pp. 105-18.

7 Parlando dal punto di vista dell'intreccio del suo romanzo, Saviane ha detto che "è nulla.... Sena e Paolo vivono la loro storia d'amore che lentamente si esaurisce con impennate sentimentali, immersioni nel paesaggio, nell'ambiente; con interventi drammatici, perfino tragici. Spunta Silva." Eppure, spiega Saviane, il suo romanzo ha una precisa finalità saggistica. "Eutanasia di un amore è tutto saggio e tutto romanzo. La mia a mbizione è proprio questa: di aver fatto un romanzo a doppia chiave di lettura: tuttavia le riflessioni che $\mathrm{i}$ fatti impongono sono ancora fatti: è un romanzo che morde la coda al saggio e questo la morde al romanzo." R. Ricchi, "Saviane: da ogni libro si puó fare un film," Nuovo cinema europeo, (ottobre 1976).

8 Spiega Saviane che nel suo romanzo Eutanasia "se la morte come valore, anzi come dolce valore e come significato è sempre presente, la morte reale è talmente lontana dal proposito del romanzo che quell'incidente lo sento adesso come un ingombro, seppur risolto, del mio bagaglio di narratore. Non che non mi piaccia, anzi è tra le pagine che amo di più, una specie di apertura sull'impossibile e sul fatale, un mistero torbido che è poi sempre la forza di propulsione del narratore." P. Ruffilli, "Tre domande a Saviane," Il Resto del Carlino, (18 aprile 1976).

9 F.A. Di Giacomo, "Preoccupa Saviane la relazione delle mamme," Il Tempo, (25 maggio 1976).

10 Intervista parzialmente inedita di Claudio Carabba, Il Castoro, 62. 